\title{
Chemical modification of Bombyx mori silk fibers with vinyl groups for thiol-ene click chemistry
}

\author{
Xiaoning Zhang ${ }^{1 *} \mathbb{D}$, Jianwei Liang ${ }^{1}$, Zhenyu Chen ${ }^{1}$, Carrie Donley ${ }^{2}$, Yuling Liu ${ }^{1}$ and Guotao Cheng ${ }^{1}$
}

\begin{abstract}
Natural Bombyx mori silk fibroin (SF) fibers were modified with 2-methacryloyloxyethyl isocyanate (MOI) first for the introduction of vinyl groups. Then, $1 \mathrm{H}, 1 \mathrm{H}, 2 \mathrm{H}, 2 \mathrm{H}$-perfluorodecanethiol was grafted onto the SF fibers via thiol-ene click chemistry using ultraviolet light. The formations of $\mathrm{MOI}$-modified and PFDT-grafted SF fibers were analyzed using Fourier transform infrared spectroscopy and X-ray photoelectron spectroscopy, respectively. The morphology of samples was also revealed by a scanning electron microscope. In addition, differential scanning calorimetry results demonstrated that SF fibers did not show significant change in thermal behavior, regardless of the chemical modification. To confirm the cytotoxicity of the prepared SF fibers, MTT [3-(4,5-dimethylthiazol-2-yl)-2,5-diphenyltetrazolium bromide] assay was performed, and no toxicity was observed with PFDT-grafted SF fibers. The results also showed that PFDTgrafted SF fibers exhibited good antifouling properties when Chlorella vulgaris (C. vulgaris) was selected as a model for algal cells adhesion experiment.
\end{abstract}

Keywords: Bombyx mori silk fibroin, Chemical modification, Thiol-ene click reaction

\section{Introduction}

Silkworm Bombyx mori can secrete silk, which consists of sericin and silk fibroin. The two paralleled fibroin fibers are held together with a layer of sericin on their surfaces [1]. Silk is a natural biopolymer and is highly appreciated for its outstanding characteristics, which majorly result from the properties and structures of silk fibroin. Unlike sericin, silk fibroin in different forms can interact with biological systems without introducing adverse immunological responses, demonstrating good biocompatibility and biodegradability [2]. Among various forms, the fibroin fibers are endowed with a combination of attractive strength, toughness, and thermal stability that surpasses many synthetic and natural fibers [3]. There is a growing interest in introducing more functionalities into silk fibroin fibers while preserving its advantageous intrinsic properties [3] in order to expand its

\footnotetext{
*Correspondence: xzhang@swu.edu.cn

1 State Key Laboratory of Silkworm Genome Biology, College of Biotechnology, Southwest University, Chongqing 400715, China

Full list of author information is available at the end of the article
}

consumption. One method, chemical modification can imbue silk fibroin fibers with new properties and functions, therefore broadening its applications in the textile and biomedical industries [4].

In 2000, Tamada et al. reported an approach to introduce a carbon double bond onto silk fibroin with 2-methacryloyloxyethyl isocyanate (MOI) [5]. Because carbon-carbon double bond can react with radicals easily, this approach allows a further modification of silk fibroin based materials, for example, polymerization with azo polymerization initiator [5]. As one example of reactions between ene functional groups and radicals, thiol-ene click reaction involves the addition of a thiyl radical across a carbon-carbon double bond in a regioand enantio-selective manner [6]. Thiol-ene click reactions are appealing for material modification because they are rapid, largely independent of solvent, in high yield, and no need to require a radical initiator $[7,8]$. To the best of our knowledge, the approach for chemical modification of SF fibers based on thiol-ene click chemistry has not been reported previously. 
Our manuscript details a technique that can modify silk fibers with thiol-containing molecules based on thiol-ene click chemistry. $1 \mathrm{H}, 1 \mathrm{H}, 2 \mathrm{H}, 2 \mathrm{H}$-perfluorodecanethiol (PFDT) was used as a model molecule as fluorine atom can serve as an elemental tag. MOI, a heterofunctional monomer that combines the versatility of a vinyl double bond and a reactive isocyanate group, was used prior to the PFDT graft. Isocyanate groups can react with hydroxyl, carboxyl, and amino side groups of amino acid residues in silk fibroin, introducing vinyl groups to silk molecules and allowing them to act as reacting points. PFDT molecules were then grafted through these reacting points onto the silk by thiol-ene click reactions. Because PFDT exhibits excellent antibioadhesive properties [9], an algal cell adhesion experiment was designed to verify the antifouling property of PFDT grafted silk and furtherly prove the successful attachment of PFDT molecules onto silk fiber surface via thiol-ene click chemistry.

\section{Methods \\ Materials}

Fresh cocoons of Bombyx mori silkworm were obtained from College of Biotechnology, Southwest University. Sodium carbonate anhydrous $\left(\mathrm{Na}_{2} \mathrm{CO}_{3}\right)$, calcium chloride anhydrous $\left(\mathrm{CaCl}_{2}\right)$, and $\mathrm{N}, \mathrm{N}$-dimethylformamide (DMF) were purchased from Kelong Chemical Reagent Factory (Chengdu, China) and at AR grade. Anhydrous ethanol $\left(\mathrm{CH}_{3} \mathrm{CH}_{2} \mathrm{OH}\right.$, AR grade) was purchased from Chongqing Chuandong Chemical Group Co., Ltd. (Chongqing, China). 1-ethyl-3-(dimethylaminopropyl) carbodiimide hydrochloride (EDC) and $N$-hydroxysuccinimide (NHS) were purchased from Solarbio (Beijing, China). Anhydrous dimethyl sulfoxide (DMSO), dibutyltin dilaurate and hydroquinone were purchased from Aladdin Industrial Co., Ltd. (Shanghai, China). 2-Isocyanatoethyl methacrylate (MOI) was purchased from Meryer Chemical Technology Co., Ltd. (Shanghai, China). $1 H, 1 H, 2 H, 2 H$-perfluorodecanethiol (PFDT) and $1 H, 1 H, 2 H, 2 H$-perfluoro-1-decanol (FTOH) were purchased from Sigma-Aldrich (Saint-Louis, USA). Ultrapure water (resistance $>18 \mathrm{M} \Omega \mathrm{cm}^{-1}$ ) was used in all experiments.

\section{Degummed silk fibroin fiber preparation}

In preparation for the experiment, Bombyx mori silkworm cocoons were cut into dime-sized pieces. To remove the sericin, those cocoon pieces were added to $500 \mathrm{ml}$ boiled sodium carbonate solution $(0.047 \mathrm{M})$ for $40 \mathrm{~min}$. Then, the degummed silk fibroin was removed from the boiling sodium carbonate solution and cooled by rinsing in ultrapure water three times for $20 \mathrm{~min}$. Each rinsing cycle was carried out in $1 \mathrm{l}$ of ultrapure water in a lab beaker, using a stir bar to circulate the volume within the beaker. After the wash, the excess water was squeezed out of the silk, and the degummed silk was then spread out on a clean piece of print paper, allowing the silk to dry in a fume hood overnight [10].

\section{Chemical modification by MOI monomer}

Once the fibroins were dry, $0.1 \mathrm{~g}$ of degummed silk fibers were immersed in $12 \mathrm{ml}$ of anhydrous DMSO under nitrogen protection, and $2.1 \mathrm{~g}$ of MOI, $2 \mu \mathrm{l}$ of hydroquinone and $0.01 \mathrm{~g}$ of dibutyltin dilaurate were added to this anhydrous system. The reaction mixture was kept at $35{ }^{\circ} \mathrm{C}$ for $24 \mathrm{~h}$. Chemically modified silk fibroin fibers were successively rinsed with DMSO, ethanol and large amounts of ultrapure water thoroughly to remove unreacted reagents and then lyophilized (LGJ-10, Shanghai YuMing Instrument Co., Ltd., China) for $24 \mathrm{~h}$.

\section{Post modification via thiol-ene click reaction}

To attach thiol-containing molecules to the MOI-modified SF fibers, each sample was contacted with $0.5 \mathrm{ml}$ of a neat PFDT liquid. A quartz coverslip $(0.5 \mathrm{~mm}$ thickness, Ted Pella) was placed on the sample to ensure homogeneous coverage of the organic liquid across the sample surface and was illuminated with a $40 \mathrm{~W}$ light-emitting diode (LED) UV lamp (UVSP1-A, ShenZhen YuXianDe Science and Technology Ltd., China) with $365 \mathrm{~nm}$ wavelength. Each sample was placed $20 \mathrm{~mm}$ below the LED UV lamp. Samples were then sonicated in ethanol to remove nonspecifically adsorbed PFDT molecules, rinsed with ultrapure water, and dried under a stream of nitrogen prior to analysis.

\section{Cultivation of algae}

Chlorella vulgaris (C. vulgaris) was obtained from Nanjing Health Biological Technology Co. Ltd. (Nanjing, China). The algae cells were cultivated for a period of 6 days, which is when the $C$. vulgaris population reached a relatively stable phase in the culture medium before the antifouling assay. Cultures were grown at $22 \pm 2{ }^{\circ} \mathrm{C}$ with $16 \mathrm{~h}$ of light exposes and an 8-h of dark photoperiod. Lighting was supplied by a combination of warm and cold fluorescent tubes, giving a luminance range of between 2200 and 2800 Lux. The in vivo absorption of the culture medium containing algal cells in each flask was monitored each day via a UV-Vis spectrophotometer (T6, Beijing Puxi Analytic Instrument Ltd., China) at $660 \mathrm{~nm}$ (the growth curve was sigmoid and is presented in Additional file 1: Figure S1).

\section{Silk fibroin fiber characterization}

$\mathrm{X}$-ray photoelectron (XP) spectroscopic characterization was carried out in an ultrahigh-vacuum system (Kratos Axis Ultra DLD, UK) with a base pressure of $5 \times 10^{-9}$ 
Torr, a monochromatic $\mathrm{Al} \mathrm{K} \alpha$ source, and a hemispherical analyzer. Survey (1.0 eV resolution) and highresolution $\left(0.1 \mathrm{eV}\right.$ resolution) spectra were collected at a $0^{\circ}$ takeoff angle from surface normal, then analyzed with Kratos Vision 2.0 software. Each high-resolution spectrum was referenced to the C1s peak $(284.6 \mathrm{eV})$ and fit with Voigt functions (70\% Gaussian, 30\% Lorentzian) after a Shirley background correction.

Scanning electron microscopy (SEM) images were acquired under the desktop SEM Phenom ${ }^{\mathrm{TM}}$ Pro (Phenom-World, Netherlands) at an accelerating voltage of $10.0 \mathrm{kV}$. Infrared spectra of samples were analyzed and recorded using Fourier transform infrared spectroscopy (FTIR, Thermo Scientific Nicolet iN10) in KBr pellets.

A differential scanning calorimeter (DSC, HSC-3, Beijing HengJiu Scientific Instrument Factory, China) utilizing $\mathrm{N}_{2}$ gas was used to assess the thermal behavior of the silk fibers under each processing condition. The scanning temperature was raised from room temperature to $390{ }^{\circ} \mathrm{C}$ at a heating rate of $10{ }^{\circ} \mathrm{C} \mathrm{min}-1$.

\section{MTT assay}

The cytotoxicity assay for PFDT-grafted SF fibers was performed against Human Embryonic Kidney 293 (HEK 293) cell-lines, a kind of epithelial cells, by MTT assay as follows [11]. After sterilization with highly compressed steam for $20 \mathrm{~min}, 0.2 \mathrm{~g}$ of sample was immersed in $10 \mathrm{ml}$ sterilized Dulbecco's modified Eagles medium (DMEM) to equilibrate at $37{ }^{\circ} \mathrm{C}$ for $72 \mathrm{~h}$. HEK 293 cells were seeded in a 96-well plate with a density of $2 \times 10^{3}$ cells/ well then incubated in $100 \mu \mathrm{l}$ of DMEM with $10 \%(\mathrm{v} / \mathrm{v})$ fetal bovine serum (FBS) for $24 \mathrm{~h}$ at $37{ }^{\circ} \mathrm{C}$ and $5 \% \mathrm{CO}_{2}$. Each well was added $10 \mu \mathrm{l}$ of leaching liquor respectively as sample group. The HEK 293 cells cultured with DMEM served as control group, and with DMEM containing $0.64 \%$ phenol served as positive group. With $24 \mathrm{~h}$, $72 \mathrm{~h}, 120 \mathrm{~h}$ incubation, $10 \mu \mathrm{l}$ of MTT solution $(5 \mathrm{mg} /$ $\mathrm{ml}$ in PBS) was added into each well respectively. After $4 \mathrm{~h}$ treatment with MTT, the medium was removed, and $200 \mu \mathrm{l}$ of DMSO was supplemented to each well immediately to dissolve the generated formazan crystal. Later, the 96-well plate was shook in a belly dancer for $15 \mathrm{~min}$ to homogenize the dissolved formazan. Quantitative detection was performed on a microplate reader (Synergy H1 Hybrid Multi-Mode Reader, Gene Company Limited) at a wavelength of $570 \mathrm{~nm}$. The cell viability was calculated according to equation:

Cell viability $(\%)=\mathrm{OD}_{570}($ sample $) / \mathrm{OD}_{570}($ control $) \times 100 \%$ where $\mathrm{OD}_{570}$ (sample) and $\mathrm{OD}_{570}$ (control) are the UV absorption of the sample and control groups at the wavelength $570 \mathrm{~nm}$, respectively.

\section{Quantification of algal cells adhering to samples}

The amount of algal cells adhering to sample was quantified from the chlorophylls released from the adherent algal cells. Briefly, non-adherent algal cells were removed following a gentle wash with $\mathrm{PBS}, \mathrm{pH}=7.4$. The sample was then immersed in $3 \mathrm{ml}$ DMSO, and placed on a rotary shaker with a speed of $150 \mathrm{rpm}$ at $30{ }^{\circ} \mathrm{C}$ for $1 \mathrm{~h}$. The extract was filtered through the $0.22 \mu \mathrm{m}$ filter membrane (Tianjin Jinteng Experimental Equipment Co., Ltd, China), and the UV absorption was recorded using UV spectrophotometry at $428.5 \mathrm{~nm}$.

\section{Results and discussion}

\section{Chemical modification by MOI monomer}

Silk fibers were chemically modified by MOI monomer in an anhydrous environment. Di- $n$-butyltin (IV) dilaurate were used as a catalyst effectively promoted the reaction between the isocyanate group and primary alcohol groups in silk fibers. The reaction was proceeded at $35^{\circ} \mathrm{C}$ based on a previously published method [5]. We plotted the weight gain in SF fibers modified with MOI monomer against the reaction time at $35{ }^{\circ} \mathrm{C}$. The mass-gain curve was sigmoid (the mass-gain curve is presented in Additional file 1: Figure S2). Weight gain was marked with increasing reaction time, finally plateauing at $20.6 \mathrm{wt} \%$ after a reaction time exceeding $24 \mathrm{~h}$. Our reaction was therefore conducted at $35^{\circ} \mathrm{C}$ for $24 \mathrm{~h} \mathrm{[5]}$.

FTIR spectra (Fig. 1) show that isocyanate and ester carbonyl peaks were clearly seen at 2270 and $1722 \mathrm{~cm}^{-1}$ in MOI monomer spectrum, respectively. The isocyanate peak in the MOI-modified silk spectrum completely disappeared after the reaction between SF and MOI, which

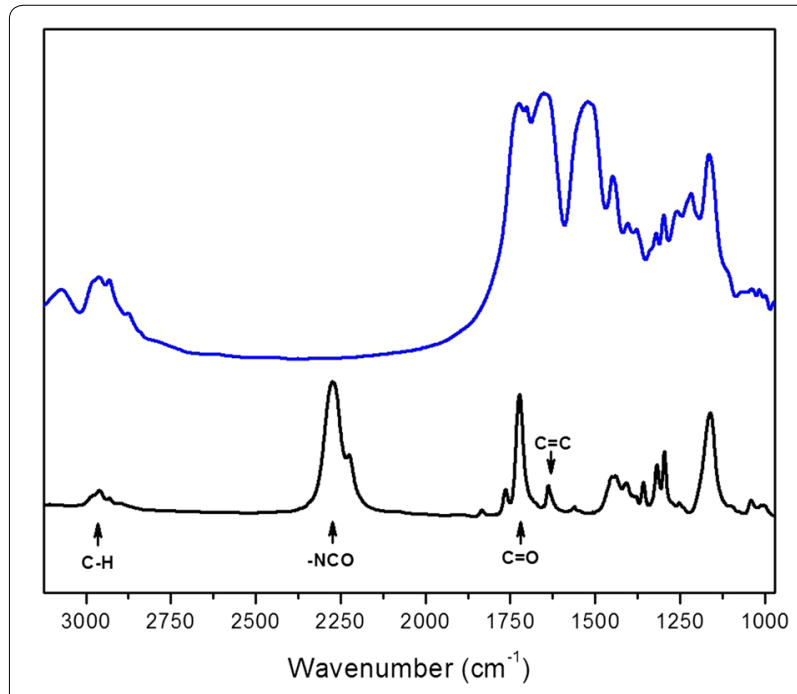

Fig. 1 FTIR spectra of MOI monomer (black line) and MOI-modified silk (blue line) 
indicates that unreactive MOI monomer did not remain on the MOI-modified silk. Thus, the chemical modification with MOI onto the silk fibers at $35^{\circ} \mathrm{C}$ was successful, although IR absorption attributed to vinyl $\mathrm{C}=\mathrm{C}$ stretching [12] could not be confirmed by FTIR analysis due to its overlapping with amide I of silk fibroin.

\section{Graft MOI-modified silk fibers with PFDT}

PFDT was grafted at vinyl groups conjugated on the MOI-modified silk fibers using a thiol-ene click reaction. To determine the selectivity of the thiol-ene click reaction, we illuminated MOI-modified silk fibers with PFDT and FTOH molecules. We chose PFDT and FTOH molecules because the fluorine atom can serve as an elemental tag. In addition, as a highly hydrophobic molecule, it is expected that PFDT molecules can provide antifouling behavior against algal cells attachment [9]. Reaction progress was tracked with XPS measurements, and the F/C ratio was used to compare the number of fluorine-containing molecules attached to the surface of each sample.

When MOI-modified silk fibers were contacted with neat PFDT and illuminated under $365 \mathrm{~nm}$ UV light for $60 \mathrm{~min}$, a fluorine peak could be detected by XPS (Fig. 2a) as a result of the thiol-ene click reaction between MOImodified silk fibers and PFDT molecules (Scheme 1), with a fluorine-to-carbon $(F / C)$ ratio of $0.03163 \pm 0.00323$. We also illuminated MOI-modified silk fibers in the presence of neat FTOH, the alcohol-containing analogue of PFDT, and we were unable to detect fluorine with XPS (Fig. 2b, $\mathrm{F} / \mathrm{C}$ ratio $=0$ ). Therefore, terminal thiol is necessary for the molecule attachment to the MOI-modified silk fibers.

To determine if PFDT attachment was specific to the MOI modification, we illuminated silk fibers without MOI modification in the presence of PFDT molecules. As a result, a 60 min illumination time resulted in no detectable fluorine electrons (Fig. 2c). The above observation
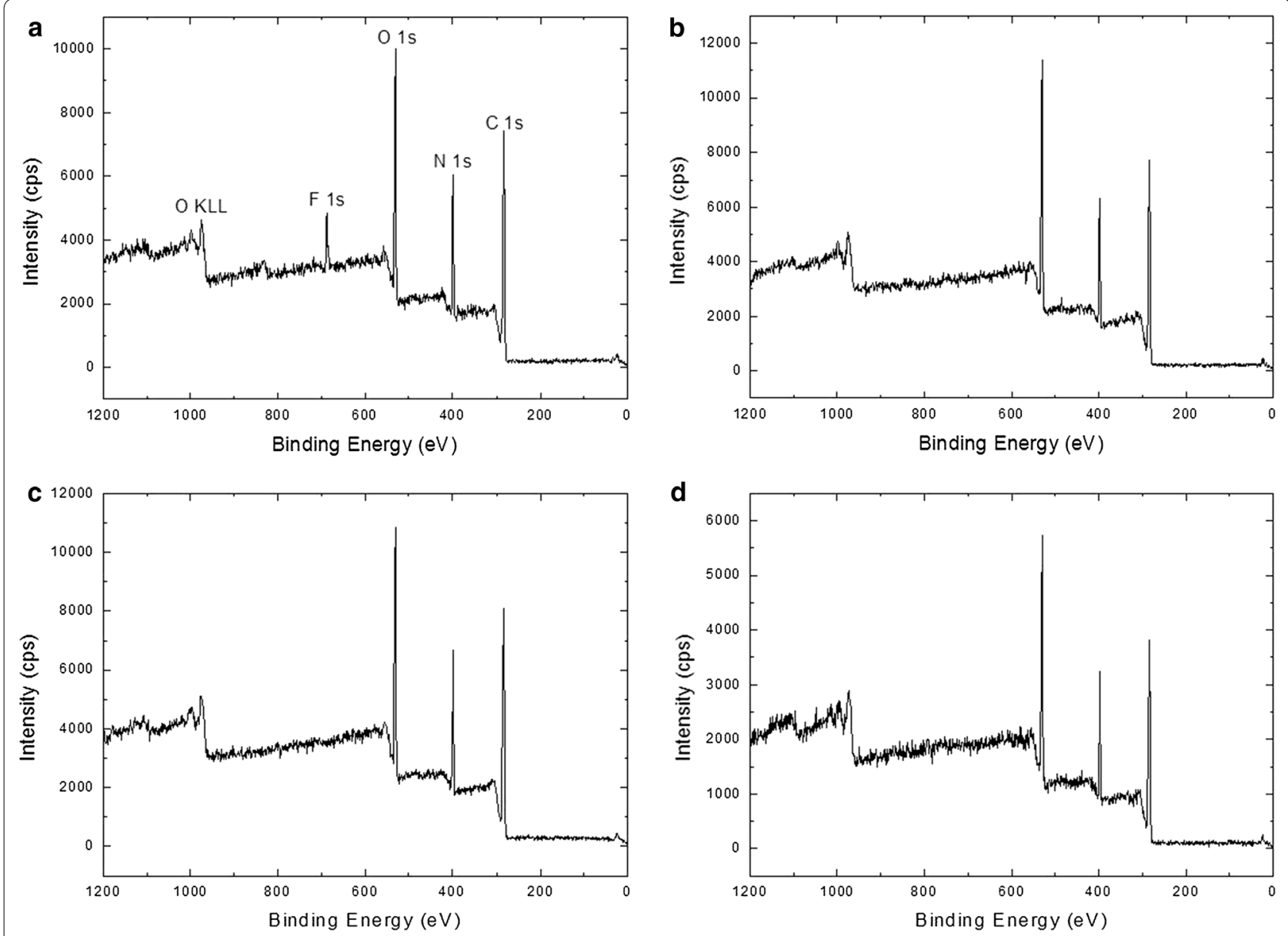

Fig. 2 Representative XP survey spectrum of MOI-modified silk with neat PFDT under 365 nm UV illumination for 60 min (a), representative XP survey spectrum of MOl-modified silk with neat PTOH under $365 \mathrm{~nm}$ UV illumination for 60 min (b), representative XP survey spectrum of original silk with neat PFDT under 365 nm UV illumination for $60 \mathrm{~min}$ (c), and representative XP survey spectrum of MOI-modified silk with neat PFDT without UV illumination (d). Binding energy assignments are as follows: O KLL: 990 eV; F1s: 686 eV; O1s: 532 eV; N1s: 400 eV; C1s: 285 eV 

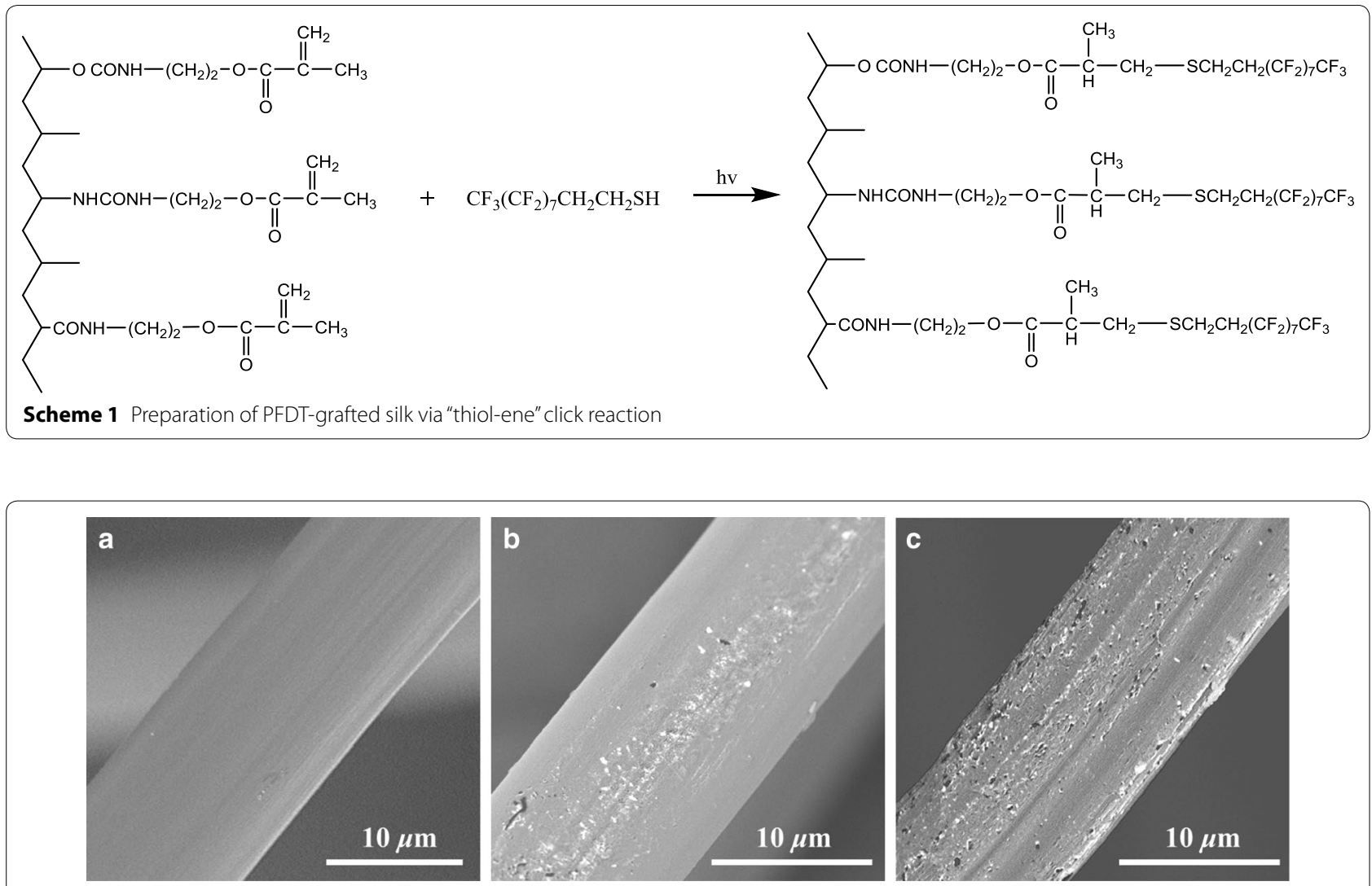

Fig. 3 SEM photograph of the original a MOI-modified, $\mathbf{b}$ and PFDT grafted, $\mathbf{c}$ SF fibers

supports the notion that an alkene group is necessary for the surface attachment through thiol-ene click chemistry.

Specifically, we incubated MOI-grafted silk fibers in the presence of PFDT molecules but in the absence of UV illumination before thoroughly rinsing and sonicating. A signal from fluorine electrons was not detectable by XPS (Fig. 2d), indicating that UV illumination of the silk fibers is required for the thiol-ene click reaction to proceed.

Figure 3 shows the SEM photograph of the original, MOI-modified, and PFDT grafted SF fibers. It seems that neither MOI nor PFDT are bonded to the SF fibers in layers. Instead, they are aggregated to form particles.

We conducted DSC measurement to investigate the thermal behavior of the original silk fibers, the MOIgrafted silk fibers, and the PFDT-grafted silk fibers (Fig. 4). The MOI-grafted silk fibers have a thermal degradation temperature of $316.5 \pm 1.0{ }^{\circ} \mathrm{C}$ (reported values are the average and standard deviation of $\mathrm{n}=3$ samples). This result indicates that the endothermic peaks of the MOI-grafted silk fibers were shifted to a lower temperature compared to that of the original silk fibers $\left(320.6 \pm 0.7{ }^{\circ} \mathrm{C}\right)$. As hydroxyl, carboxyl, and amino side groups of amino acid residues in silk fibroin would be replaced by MOI molecules, which are methyl and

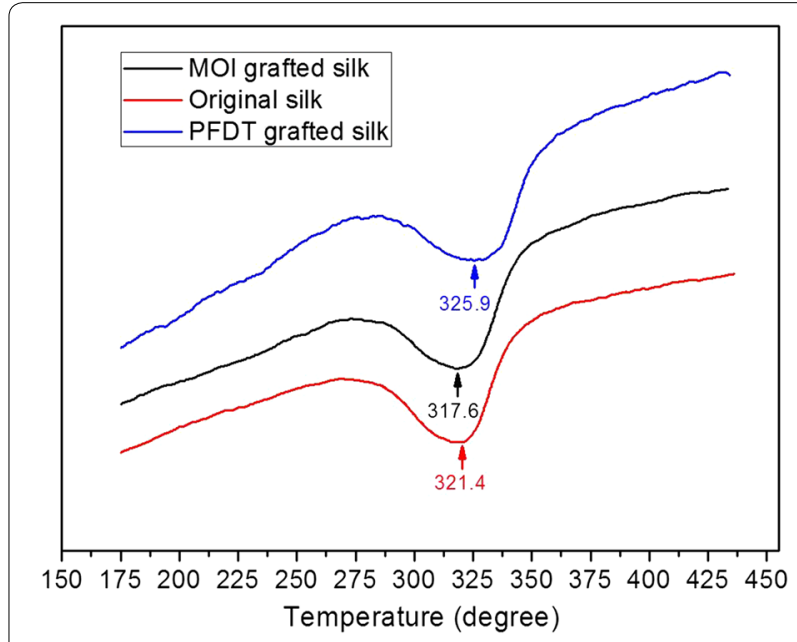

Fig. 4 Representative DSC curves of original silk fibers, MOI-modified silk fibers, and PFDT-grafted silk fibers with UV light illumination for $60 \mathrm{~min}$

vinyl-terminated, it is estimated that the original intermolecular interactions-such as hydrogen bonds-would be disrupted, therefore resulting in decreased thermal 
stability of silk fibers. While the DSC curves of PFDTgrafted silk fibers have a thermal degradation temperature of $324.9 \pm 0.9{ }^{\circ} \mathrm{C}$, which shows a shift to higher values compared to the thermal degradation temperature of the original ones. As fluorine-containing molecules have superior thermal stability [13], this behavior can be explained by successful PFDT grafting onto the silk fibers. In summary, although thermal degradation temperatures of silk fibers shifted upon chemical modification at each step, the samples did not show any significant change in their thermal behaviors.

\section{Cell viability assay}

To investigate the toxicity profile of the PFDT-grafted silk fibers, the standard MTT cytotoxicity assay [14] with Human Embryonic Kidney 293 (HEK 293) cells was performed. The cells were treated with leaching liquor from PFDT-grafted silk fibers. The cell viability of the samples were higher than that of control group, suggesting PFDTgrafted silk fiber exhibited high biocompatibility, which is the essential characteristic for biomaterials (Fig. 5).

\section{Algal cells adhesion experiment}

To evaluate the antifouling performance of PFDT-grafted silk fibers and confirm the successful binding of PFDT on the surface of silk fibers via thiol-ene click chemistry, we immersed the PFDT-grafted silk fibers in a C. vulgaris culture for 1 week and 2 weeks, respectively. The cellcultured samples were rinsed with a phosphate-buffered solution (PBS, $\mathrm{pH}=7.4$ ) three times to remove all nonadherent cells. The samples were then consecutively immersed in DMSO to extract chlorophyll, as described above. The amount of cells adhered to the samples was evaluated with UV spectrophotometry at a wavelength of $428.5 \mathrm{~nm}$ by measuring the chlorophyll content.

It was revealed that the PFDT-grafted silk fibers demonstrated less cell adhesion over both 1-week and 2-week algal cell culture periods (Fig. 6). In addition, although the amount of $C$. vulgaris cells adhered to original silk fibers increased as the increased incubation time, cells adhered to the PFDT-grafted silk fibers remain stable. Above results demonstrate good antifouling properties of the PFDT-grafted silk fibers and indicate the importance of surface chemical structure changes of silk fibers in preventing algal cell adhesion. PFDT incorporation changed algal cell adhesion because the hydrophobic moiety was introduced onto the surface of the silk fibers.

\section{Conclusion}

In this work, we installed vinyl groups onto the silk fibroin fibers. Then, PFDT, as a model molecule, was grafted onto silk fibers using a thiol-ene click reaction in a heterogeneous system. Through different experimental designs, we specifically determined that (i) a thiol group of the functional molecule is required for attachment; (ii) vinyl groups on the silk fibers are needed for molecule attachment; and (iii) UV illumination of the sample is necessary for the reaction to proceed. C. vulgaris adhesion significantly decreased on PFDT-grafted silk fiber compared to original silk fibers. This result indicates that antifouling property can be lent to silk, and a thiol-ene click reaction initiated by UV illumination provides a method of grafting functional molecules onto silk fibers. This work could provide new perspectives for the application of the chemical modification techniques for silk fibroin and will also expand the spectrum of silk

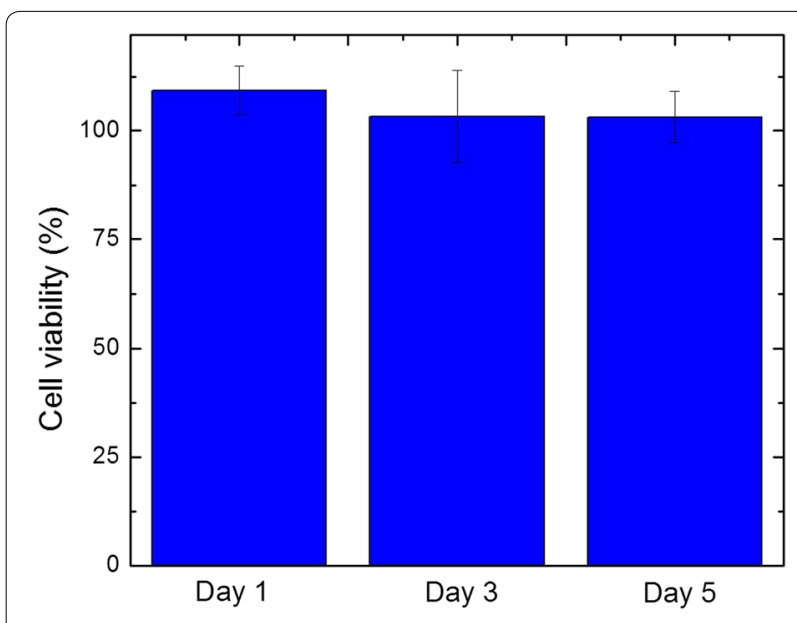

Fig. 5 In vitro viability toward HEK 293 cells after incubation with leaching liquor from PFDT-grafted silk fibers for 24 h, 72 h, and $120 \mathrm{~h}$ respectively

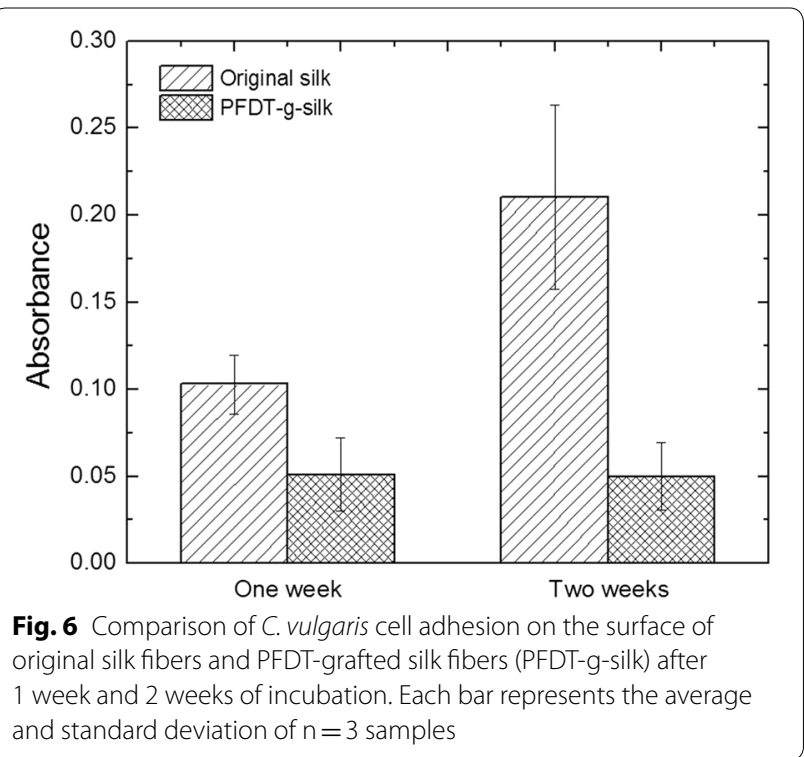


utilization. We believe that further reaction optimization (e.g. design a light source which could illuminate silk fibers with UV light evenly) would realize a controlled graft density of the functional molecules on the silk fibers, therefore facilitating the alternation of chemical and physical properties of silk fibers.

\section{Supplementary information}

Supplementary information accompanies this paper at https://doi. org/10.1186/s13065-019-0630-7.

Additional file 1: Figure S1. Optical density of the Chlorella vulgaris culture at $660 \mathrm{~nm}$ as a function of time. Figure S2. Weight gain of $\mathrm{MO}$ monomer on SF fibers as a function of reaction time. The weight gain of SF fibers after $\mathrm{MOI}$ modification was calculated as follows: weight gain $(w t \%)=100 \times\left(W_{2}-W_{1}\right) / W_{1}$, where $W_{1}$ and $W_{2}$ are the dried original SF fibers and MOl-modified SF fibers, respectively.

\section{Abbreviations}

SF: silk fibroin; MOI: 2-methacryloyloxyethyl isocyanate; PFDT: $1 H, 1 H, 2 H, 2 H-$ perfluorodecanethiol; FTOH: 1H,1H,2H,2H-perfluoro-1-decanol; XPS: X-ray photoelectron spectroscopies; FTIR: Fourier transform infrared; SEM: scanning electron microscopy; DSC: differential scanning calorimeter; DMF: N,Ndimethylformamide; DMSO: anhydrous dimethyl sulfoxide; EDC: 1-ethyl-3(dimethylaminopropyl) carbodiimide hydrochloride; NHS: N-hydroxysuccinimide; C. vulgaris: Chlorella vulgaris; LED: light-emitting diode.

\section{Authors' contributions}

XZ designed and conducted the experiments related to this study and wrote the paper. $\mathrm{JL}$ and $\mathrm{YL}$ prepared resources for the experiments and collected the data for algal cells adhesion experiment. CD collected the XPS data. ZC performed measurements on DSC. GC reviewed and edited this manuscript. All authors read and approved the final manuscript.

\section{Funding}

This research was funded by Fundamental Research Funds for the Central Universities (XDJK2018C067), Chongqing Municipal Commission of Commerce (CQ2019JSCC03) and a Start-up Fund of Southwest University Grant (SWU117036). This work was performed in part at the Chapel Hill Analytical and Nanofabrication Laboratory, CHANL, a member of the North Carolina Research Triangle Nanotechnology Network, RTNN, which is supported by the National Science Foundation, Grant ECCS-1542015, as part of the National Nanotechnology Coordinated Infrastructure, NNCl. This work was supported in part by the Chongqing Graduate Student Research Innovation Project (CYS19121).

\section{Availability of data and materials}

The datasets used and analysed during the current study are available from the corresponding author on reasonable request.

\section{Competing interests}

The authors declare that they have no competing interests.

\author{
Author details \\ 1 State Key Laboratory of Silkworm Genome Biology, College of Biotechnol- \\ ogy, Southwest University, Chongqing 400715, China. ${ }^{2}$ Chapel Hill Analytical
}

and Nanofabrication Laboratory, Department of Applied Physical Sciences, University of North Carolina at Chapel Hill, Chapel Hill, NC 27599-3216, USA.

Received: 11 May 2019 Accepted: 31 August 2019

Published online: 10 September 2019

\section{References}

1. Qi Y, Wang H, Wei K, Yang Y, Zheng R-Y, Kim I et al (2017) A review of structure construction of silk fibroin biomaterials from single structures to multi-level structures. Int J Mol Sci 18(3):237

2. Wang Z, Zhang Y, Zhang J, Huang L, Liu J, Li Y et al (2014) Exploring natural silk protein sericin for regenerative medicine: an injectable, photoluminescent, cell-adhesive 3D hydrogel. Sci Rep 4:7064

3. Koh L-D, Cheng Y, Teng C-P, Khin Y-W, Loh X-J, Tee S-Y et al (2015) StruCtures, mechanical properties and applications of silk fibroin materials. Prog Polym Sci 46:86-110

4. Huang W, Ling S, Li C, Omenetto FG, Kaplan DL (2018) Silkworm silkbased materials and devices generated using bio-nanotechnology. Chem Soc Rev 47(17):6486-6504

5. Furuzono T, Ishihara K, Nakabayashi N, Tamada Y (2000) Chemical modification of silk fibroin with 2-methacryloyloxyethyl phosphorylcholine. II. Graft-polymerization onto fabric through 2-methacryloyloxyethyl isocyanate and interaction between fabric and platelets. Biomaterials 21(4):327-333

6. McKenas CG, Fehr JM, Liu B, Donley CL, Lockett MR (2018) Mechanistic insights into UV-initiated thiol-ene reactions on amorphous carbon films. J Phys Chem C 122(38):21854-21860

7. McKenas CG, Fehr JM, Donley CL, Lockett MR (2016) Thiol-ene modified amorphous carbon substrates: surface patterning and chemically modified electrode preparation. Langmuir 32(41):10529-10536

8. Hoyle CE, Bowman CN (2010) Thiol-ene click chemistry. Angew Chem Int Ed 49(9):1540-1573

9. Fan L, Li B, Zhang J (2015) Antibioadhesive superhydrophobic syringe needles inspired by mussels and lotus leafs. Adv Mater Interfaces 2(8):1500019

10. Zhang X, Bao H, Donley C, Liang J, Yang S, Xu S (2019) Thiolation and characterization of regenerated Bombyx mori silk fibroin films with reduced glutathione. BMC Chem 13(1):62

11. Ye D, Zhong Z, Xu H, Chang C, Yang Z, Wang Y et al (2016) Construction of cellulose/nanosilver sponge materials and their antibacterial activities for infected wounds healing. Cellulose 23(1):1-15

12. Smulevich G, Miller MA, Kraut J, Spiro TG (1991) Conformational change and histidine control of heme chemistry in cytochrome c peroxidase: resonance Raman evidence from Leu-52 and Gly-181 mutants of cytochrome c peroxidase. Biochemistry 30(39):9546-9558

13. Knight GJ, Wright WW (1982) Thermal degradation of some fluorinecontaining elastomers. Polym Degrad Stab 4(6):465-478

14. Supino R (1995) MTT assays. Methods Mol Biol 43:137-149

\section{Publisher's Note}

Springer Nature remains neutral with regard to jurisdictional claims in published maps and institutional affiliations. 